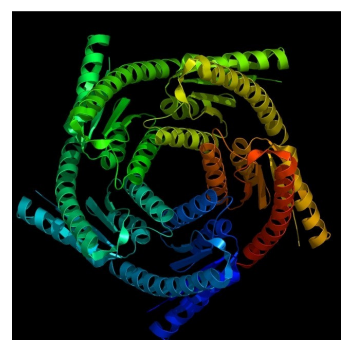

\title{
Crystallization and Characterization of Galdieria sulphuraria RUBISCO in Two Crystal Forms: Structural Phase Transition Observed in P2 ${ }_{1}$ Crystal Form
}

\author{
Michael Baranowski ${ }^{1}$ and Boguslaw Stec ${ }^{2, *}$
}

1 Department of Chemistry, University of Texas at El Paso, 500 W. University Ave. El Paso, TX 79968, USA

2 Burnham Institute for Medical Research, 10901 N. Torrey Pines Rd. La Jolla, CA 92037, USA

* Author to whom correspondence should be addressed; E-mail: bstec@burnham.org

Received: 13 July 2007; in revised form: 15 October 2007 / Accepted: 16 October 2007 /

Published: 23 October 2007

\begin{abstract}
We have isolated ribulose-1,5-bisphosphate-carboxylase/oxygenase (RUBISCO) from the red algae Galdieria Sulphuraria. The protein crystallized in two different crystal forms, the I422 crystal form being obtained from high salt and the $\mathrm{P} 2{ }_{1}$ crystal form being obtained from lower concentration of salt and PEG. We report here the crystallization, preliminary stages of structure determination and the detection of the structural phase transition in the $\mathrm{P} 2{ }_{1}$ crystal form of $G$. sulphuraria RUBISCO. This red algae enzyme belongs to the hexadecameric class (L8S8) with an approximate molecular weight 0.6MDa. The phase transition in G. sulphuraria RUBISCO leads from two hexadecamers to a single hexadecamer per asymmetric unit. The preservation of diffraction power in a phase transition for such a large macromolecule is rare.
\end{abstract}

Keywords: RUBISCO, red algae, crystallization, phase transition. 


\section{Introduction}

In eukaryotic photosynthetic organisms, $\mathrm{CO}_{2}$ assimilation (fixation) is carried out by a series of biochemical reactions (the Calvin cycle) that take place in chloroplasts [1]. This biochemical cycle is subdivided into three stages. In stage one, $\mathrm{a} \mathrm{CO}_{2}$ molecule is incorporated into a five-carbon backbone of a ribulose-1,5-bisphosphate that is cleaved into two molecules of phosphoglyceric acid. In stage two, three carbon backbone moieties, created in stage one, are handled and streamlined into other biochemical pathways. In stage three, a pentacarbon substrate is regenerated. In essence, the whole act of $\mathrm{CO}_{2}$ incorporation is carried out in stage one by the enzyme ribulose-1,5-bisphosphate carboxylase/oxygenase (RUBISCO, E.C. 4.1.1.39). This enzyme is one of the most abundant proteins in our biosphere, and it is mostly responsible for the biomass creation. It catalyzes the carboxylation reaction in which the $\mathrm{CO}_{2}$ is added to the $\mathrm{C} 2$ of ribulose-1,5-bisphosphate. Subsequently, the hexacarbon intermediate is cleaved forming two 3-phosphoglycerates [2]. These simple sugars can be utilized in several ways including glycolysis (energy production), storage (starch production) or regeneration of the initial substrate ribulose-1,5-bisphosphate. The enzyme can also incorporate $\mathrm{O}_{2}$ and produce 3-phosphoglycerate and 2-phosphoglycolate. This second reaction is considered parasitic and diminishes the overall rate of $\mathrm{CO}_{2}$ fixation. This particular species of algae has a high specificity ratio for the $\mathrm{CO}_{2} / \mathrm{O}_{2}$ incorporation and should provide insights into the molecular origin of specificity and slow catalytic turnover rate.

RUBISCO from higher plants, as well as from cyanobacteria, red algae and some photosynthetic bacteria, forms a hexadecameric structure, built out of eight large and eight small subunits. The molecular weight of a large subunit is around $55 \mathrm{kDa}$, while a small subunit is $\sim 16 \mathrm{kDa}$. The threedimensional structure of RUBISCO's from several species of plants, as well as from more primitive organisms, have been elucidated by X-ray crystallography. The large subunit containing the active site is folded into the $\alpha / \beta$ (TIM) barrel, and the small subunit has an $\alpha+\beta$ fold. The small subunit seems to play mostly a structural role in the assembly of this large molecule, although recent reports have also suggested some catalytic role, especially in the selectivity towards the $\mathrm{CO}_{2}$. The genes for both subunits of $G$. sulphuraria RUBISCO are coded by the chloroplast DNA. This is in contrast to RUBISCO from plants (e.g. tobacco) where the gene for small subunit is usually coded by the nuclear DNA. Its nuclear location in most species is probably the reason for a higher sequence divergence of the small subunit, especially when compared to that from higher plants.

$\mathrm{X}$-ray crystallography provides a wealth of structural information about the different stages of RUBISCO catalysis. Structures of the unactivated enzyme [3], the activated one with metal ions and transition state analog [4], the structures with substrate analogs [5] as well as products are available in the PDB. A few years ago, the structure of RUBISCO from a similar species G. partita was solved and published [6]. However, structural details of activation were not fully described nor the catalytic cycle fully elucidated [7].

In order for the enzyme to carry out its catalytic mission, it needs to be activated by addition of one molecule of $\mathrm{CO}_{2}$ to a crucial Lys residue. The active site Lys must be carbamylated to provide an essential ligand for binding of the metal ion. This activation process in plants is mediated by RUBISCO activase [8] that facilitates the release of blocking agents. It may also happen nonenzymatically, by the prolonged exposure to higher levels of $\mathrm{CO}_{2}$. In an activated enzyme, the 
substrate is directly coordinated to the metal ion. It is believed that the second $\mathrm{CO}_{2}$ molecule is transiently coordinated to the metal ion. In the proposed reaction, the metal ion provides necessary polarization to activate the $\mathrm{CO}_{2}$ molecule, while the carbamyl moiety plays the role of a base in the reaction. The catalytic mechanism was investigated by a number of biochemical methods such as: isotope exchange, NMR and fast kinetics, as well as by computational methods. The results were summarized by several reviews; one notable that summarizes the kinetic mechanism is by Cleland $e t$. al. [7].

The red algae G. sulphuraria (previously known as Cyanidium caldarium, Allen strain) appears to be an old organism with a large capacity to adapt to different environments. It can be found in almost every hot spring around the globe and can grow under very diverse conditions that include significantly changed gaseous environments (anaerobic) and varied temperatures $\left(20-50^{\circ} \mathrm{C}\right)$. It is an extremophile that prefers acidic conditions $(\mathrm{pH} \sim 2)$ and achieves optimum growth at $42^{\circ} \mathrm{C}$, but can dwell at $\mathrm{pH}=0$ and higher temperatures [9].

The large subunit of RUBISCO from red algae G. sulphuraria has 493 amino acids while the small subunit has 138 amino acids [10]. The structure of RUBISCO from this species is interesting since the attempts to reengineer the specificity of RUBISCO towards a more selective enzyme that would suppress or eliminate the oxygenase activity have been unsuccessful [11]. The red algae may hold great promise in environmental remediation, especially in the era of global warming caused by green-house gasses (e.g. $\mathrm{CO}_{2}$ ), because red algae RUBISCO was proven to have the highest specificity toward $\mathrm{CO}_{2}$ [12].

\section{Results and Discussion}

\subsection{Structure solution and the architecture}

We purified G. sulphuraria RUBISCO from algae cultured in the laboratory following the published procedures [13]. The protein proved to be heat stable and structurally stable under refrigerated conditions. It was possible to crystallize the enzyme from samples that were stored at $4^{\circ} \mathrm{C}$ for a few years after purification. Large crystals of an unusual lens-like morphology were obtained from the high salt condition at $\mathrm{pH} \sim 7$. The crystals diffracted to $\sim 2 \AA$ resolution and the diffraction pattern suggested the I422 space group as seen before in other RUBISCO crystals [14]. The structure was solved by the molecular replacement technique using the structure of spinach RUBISCO as a probe model. The spinach RUBISCO was used because the crystals for the form 1 were obtained and their structure solution was carried out, before the structure of RUBISCO from a G. partita was published and available. RUBISCO from the red algae $G$. sulphuraria. belongs to the hexadecameric class (L8S8), and the approximate molecular weight of the entire assembly is $\sim 0.6 \mathrm{MDa}$. The general organization of the enzyme is very similar to that observed for other hexadecameric RUBISCO structures from plants. The asymmetric unit contained large and small subunits of RUBISCO. The entire hexadecameric molecule is obtained by application of the crystal symmetry. The hexadecamer was positioned in such a manner that the internal 422 symmetry coincided with that of the lattice. Such an arrangement resulted in neighboring hexadecamers being aligned. 
The initial solution indicated that the small subunit architecture was significantly different from that of higher plants. That observation agrees with the genetic data indicating that the small subunit of this species is also coded by the chloroplast DNA, in opposition to plants in which the small subunit is coded by the nuclear DNA. When eventually RUBISCO from G. partita became available, it turned out that the small subunit architecture is very similar in this closely related species. The sequence deviation of G. sulphuraria from G. partita is low ( $97 \%$ identity in the large subunit and $90 \%$ identity in the small subunit). The extended $\beta$-sheet of the small subunit, not present in higher plants RUBISCO, forms an eight-stranded beta-barrel around a fourfold axis [15].

Figure 1. Images presenting morphology for both crystal forms: (A) crystal form 1 (I422), (B) crystal form 2 (P2 $2_{1}$.

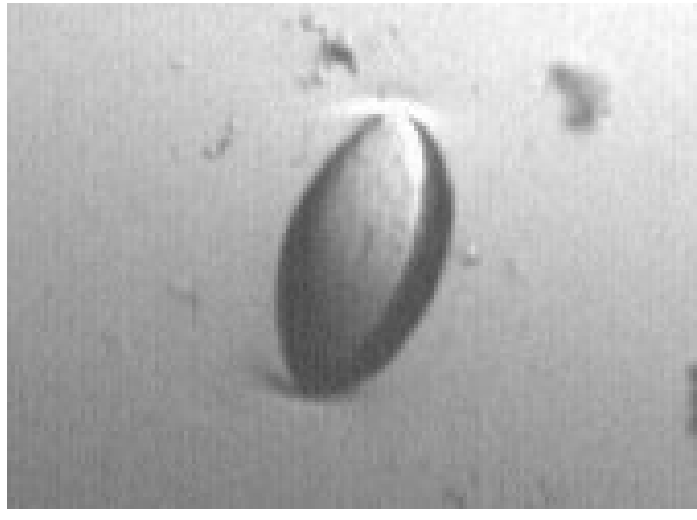

A

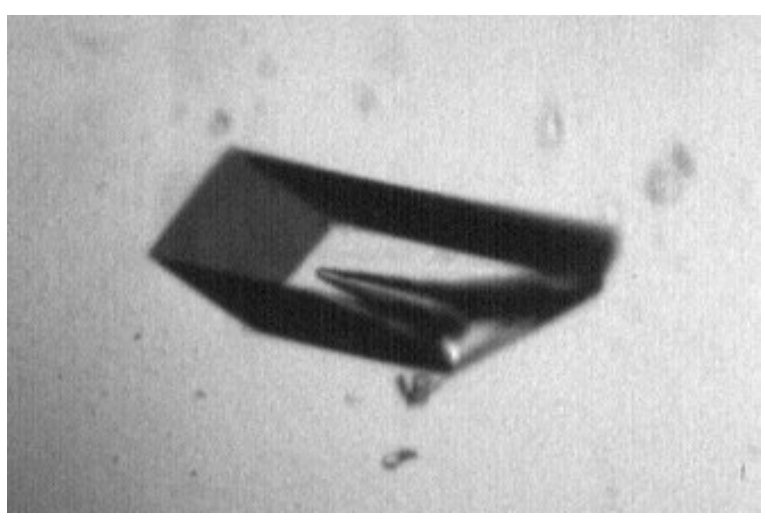

B

In our search for better conditions to activate RUBISCO, we initiated new crystallization trials that resulted in the second crystal form. This crystal form was obtained from a lower ionic strength condition, with PEG 4000 as a precipitant. Large rectangular prism crystals (maximal dimension $\sim 0.7$ $\mathrm{mm}$ ) showed a monoclinic system with the $\mathrm{P} 2{ }_{1}$ space group. Apparently, the asymmetric unit contained the entire hexadecamer. The difference in morphology between the I422 and P2 $2_{1}$ crystal forms can be seen in Figure 1. An independent view of each active site in the hexadecamer of this new crystal form (unaveraged by internal symmetries as it happens in the crystal form 1) might allow for a more detailed observation of changes upon the activation process in the crystal.

\subsection{Structural phase transition in the $P 2{ }_{1}$ crystal form}

The diffraction images of the monoclinic crystal showed a strong ring-like pattern, characteristic of imperfect glassification of solvent. To clear the diffraction images from this artifact, we took the frozen crystal, bathed it in fresh cryoprotecting buffer and froze it again in an $\mathrm{N}_{2}$ stream. The single crystal diffraction pattern was obtained, but with visible changes. The diffraction pattern before and after the freeze-thaw cycle is presented in Figure 2. The changes in diffraction pattern upon data collection and successful indexing convinced us that we detected the structural phase transition. Presently, all three structures are undergoing detailed refinements, the results of which will be published separately. 
Figure 2. Representative examples of diffraction images for both $\mathrm{P} 2_{1}$ crystal forms: (A) crystal form $2 \mathrm{a},(\mathrm{B})$ crystal form $2 \mathrm{~b}$. The resolution at the edge of the frame corresponds to $\mathrm{d} \sim 2 \AA$. The maximal resolution of form $2 \mathrm{a}$ extends to $\sim 2.4 \AA$, while for form $2 \mathrm{~b}$ to $\sim 2.7 \AA$. The insets show the arrangement of the lattices and the approximate relationships between the reciprocal distances.

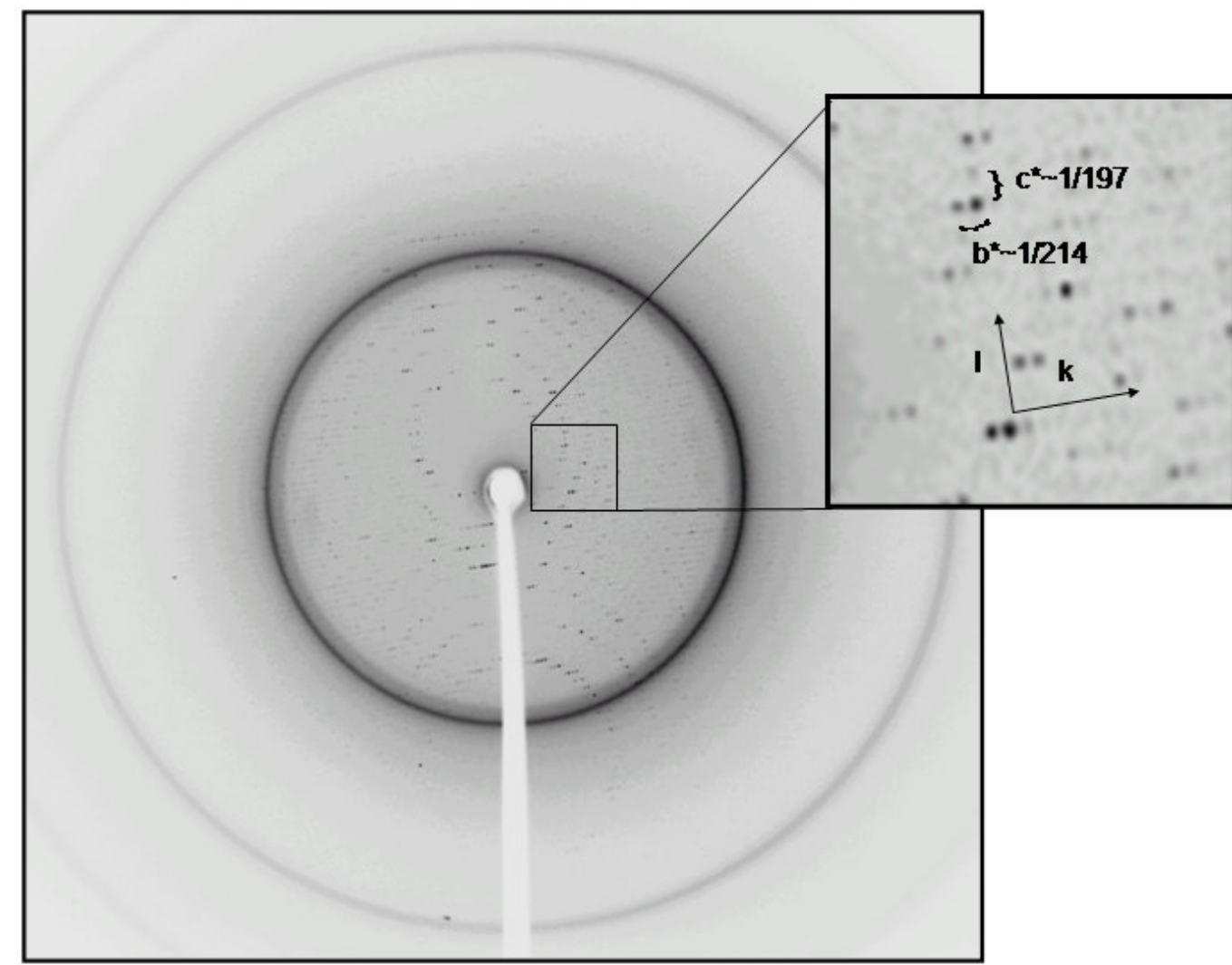

A

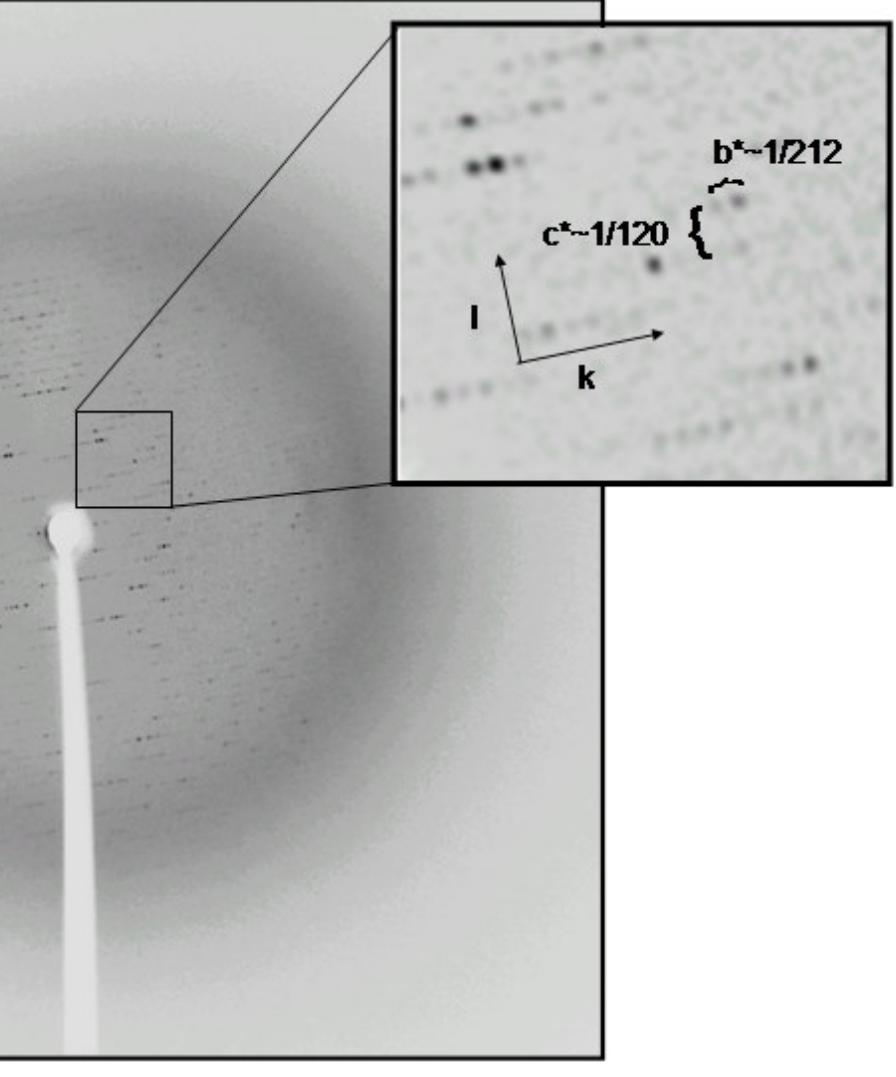


We focus here on the differences between different crystal forms. In the I422 crystal form, the hexadecamers were tightly aligned with side to side contacts resulting in $39.2 \%$ of solvent content (assuming the molecular weight of the small and the large subunit $71193 \mathrm{Da}$ ). In both P2 $2_{1}$ crystal forms the hexadecamers were staggered, which led to a much higher solvent content of $\sim 47 \%$. We inferred that the phase transition in the $\mathrm{P} 2{ }_{1}$ crystal form was associated with the changes related to thawing, bathing in fresh cryoprotectant and freezing again. The differences in packing and the postulated relationships between the lattices are shown in Figures 3, 4 and 5. Figure 3 represents steroscopic projections of the self-rotation function. While the pattern in Figure $3 \mathrm{~A}$ is self-evident, with internal symmetry elements overlapping with the crystal symmetry of the I422 space group, the interpretation of plots in $3 \mathrm{~B}$ and $\mathrm{C}$ is more complex. Figure 5 helps in correlating the crystallographic $\mathrm{X}$ and $\mathrm{Z}$ directions with that of internal symmetries of the assembly in different crystal forms. Figure 3B confirms the deviation of the twofold axis of the hexadecamer from the $\mathrm{X}$ direction by around $20^{\circ}$, while figure $3 \mathrm{C}$ shows an almost perfect alignment of the twofold axis with the $\mathrm{X}$ direction.

Figure 3. Plot of self-rotation function for all crystal forms of RUBISCO: (A) crystal form 1, (B) crystal form 2a, (C) crystal form $2 \mathrm{~b}$. Please note the presence of dominant features coinciding with crystallographic axes in the highly symmetric I422 crystal form, and the presence of multiple off-axis symmetry elements in lower symmetry $\mathrm{P} 2{ }_{1}$ crystal form. The peaks indicate the change of the direction of the $a$ axis in different forms and suggesting the alignment of hexadecamers in form $2 \mathrm{~b}$.

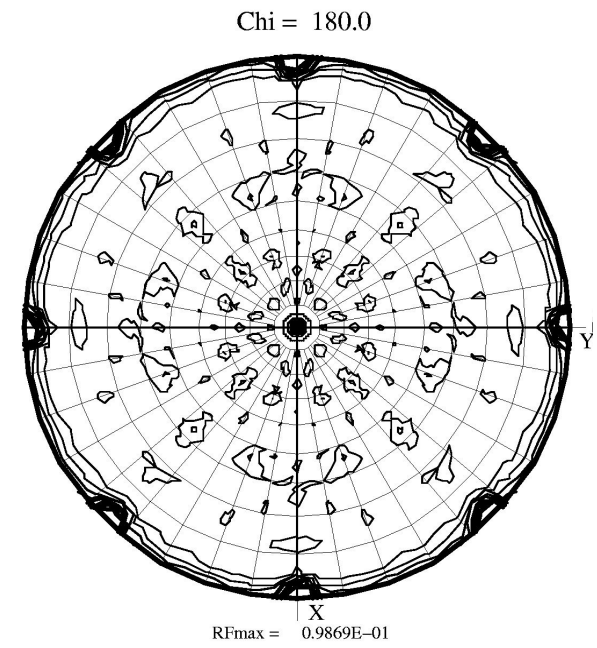

A

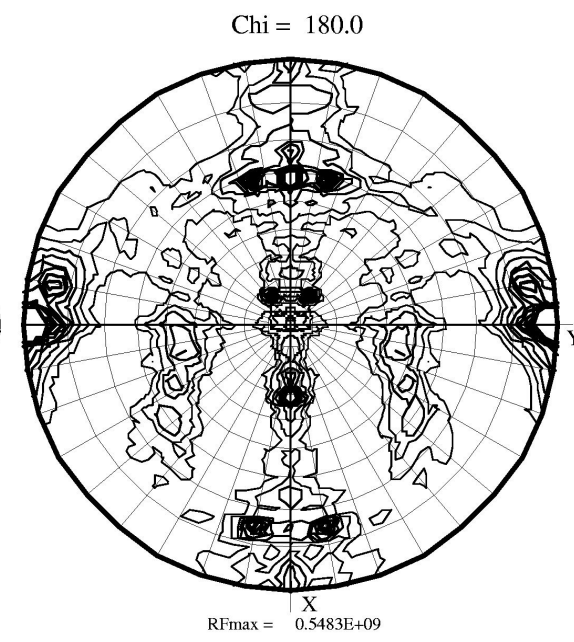

B

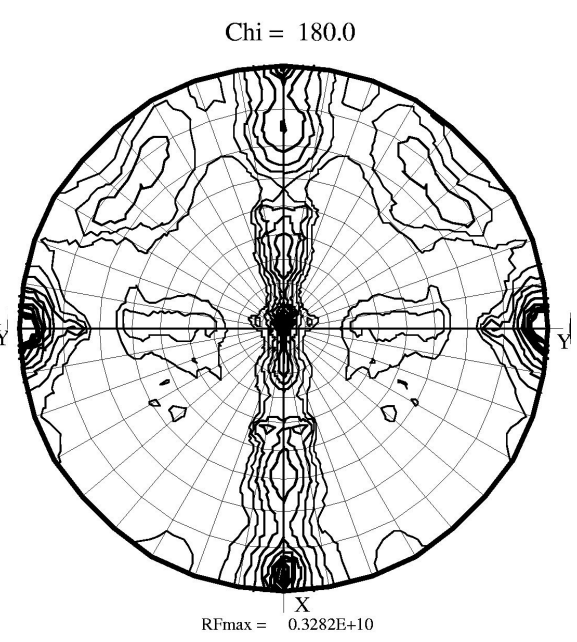

C

After having solved the phase problem, we inspected the models in both $\mathrm{P} 2{ }_{1}$ crystal forms. Our cursory inspection showed that the centers of gravity of the hexadecamers did not change noticeably. Apparently, the hexadecamers in form $2 b$ rotated in such a manner that they became aligned against the major crystal axis, and their four-fold axes lined up approximately with the Y direction. This fact led to the reduction of dimensions of the crystal lattice. Noticeable is a small reduction in the $b$ axis while the relationship between the $a$ and $c$ axes stays in proportion to the relationship of the surfaces spanned by the $a, c$ vectors. According to the construction presented in Figure 5, the surface of the $a c$ face of the new cell should be approximately half of the old cell, what is also equivalent to that the $a^{\prime}$ and $c^{\prime}$ are approximately equal to half-diagonals of the $a c$-face of the original cell. This relationship can be 
expressed as a cross product of the axc vectors, that can be further expressed in scalar values as a c $\sin \beta \approx 2$ a' $\mathrm{c}^{\prime} \sin \beta$ '. The global change in packing leads to the $\sim 1 \%$ reduction in the solvent content.

The difference between the packing schemes is not only in the presence of the second hexadecamer in the asymmetric unit, but also in mutual positioning of the nearest neighbors. We present the difference in the general packing models in Figure 4. The analysis of both crystal forms showed that the second hexadecamer underwent a small rotation $\left(\sim 3^{\circ}\right)$ around the axis positioned in the $a b$ plane. The change is best visualized by superposition of two independent hexadecamers of form $2 \mathrm{a}$ onto the single hexadecamer of form $2 \mathrm{~b}$ (Figure $4 \mathrm{~d}$ ). The effective rotation of the second hexadecamer by $\sim 3^{\circ}$ results in aligning the molecules what leads to a smaller unit cell and reduced contents of the asymmetric unit.

Figure 4. Packing diagrams for both crystal forms of RUBISCO: (A) I422 crystal form (large and small subunit in yellow) (B) $\mathrm{P} 2{ }_{1}$ crystal form $2 \mathrm{a}$, (C) $\mathrm{P} 2{ }_{1}$ crystal form $2 \mathrm{~b}$. Please note similarities in packing, despite the difference in the cell dimensions and contents of the asymmetric unit (hexadecamers in yellow). (D) Comparison of both crystal lattices represented by superposition of the two independent hexadecamers of the

form $2 \mathrm{a}$ (in purple) onto the single hexadecamer of form $2 \mathrm{~b}$ (yellow). The effective rotation of the second hexadecamer by $\sim 3^{\circ}$ results in aligning them and the reduction of the asymmetric unit.

A

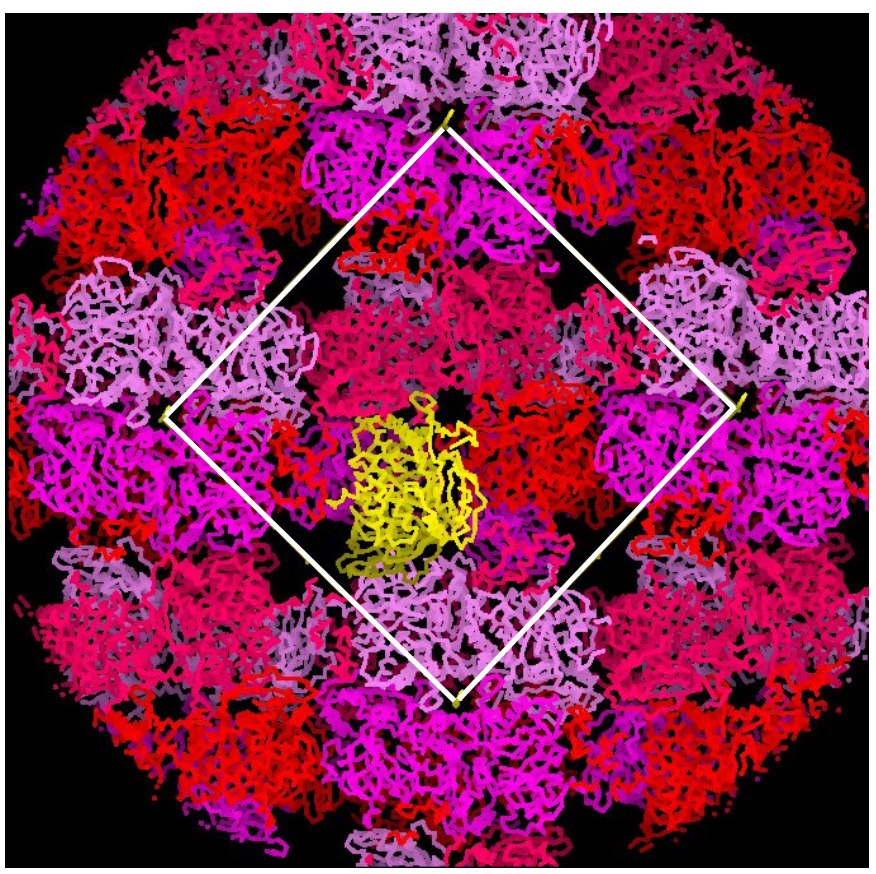

B

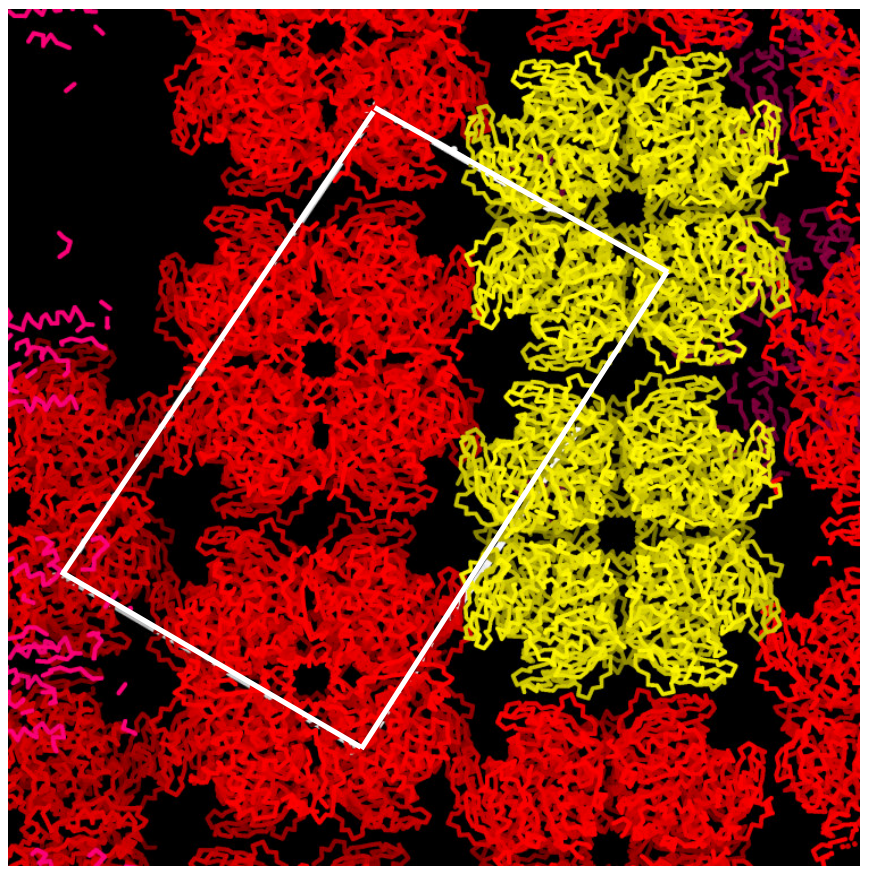


Figure 4. Cont.

C

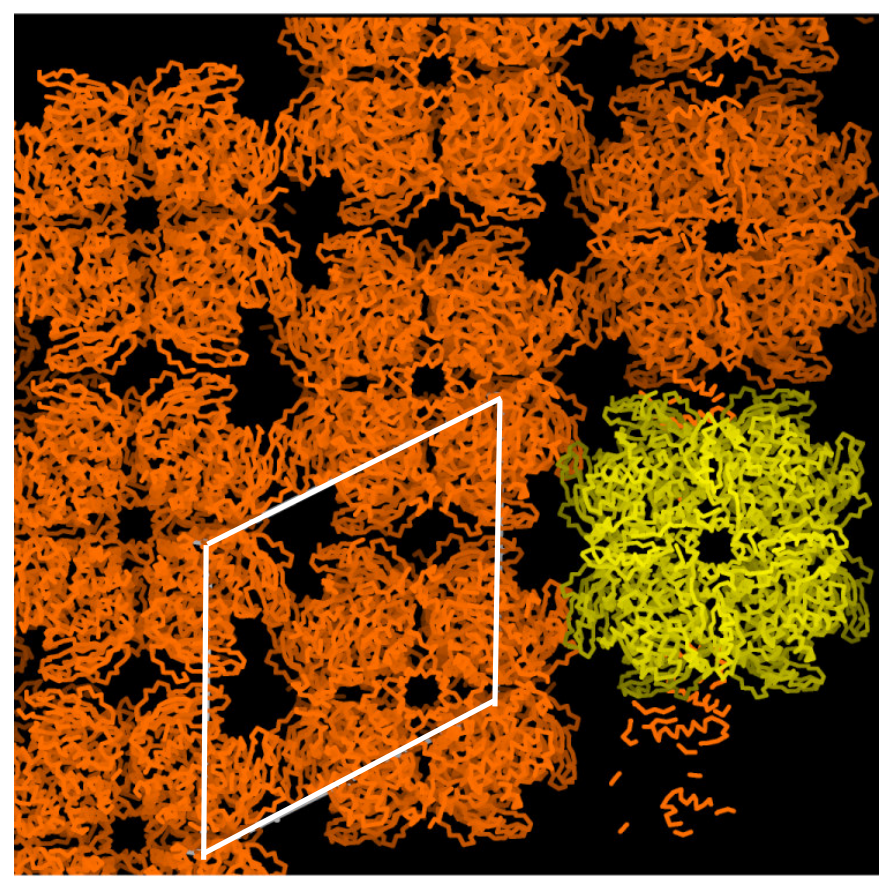

D

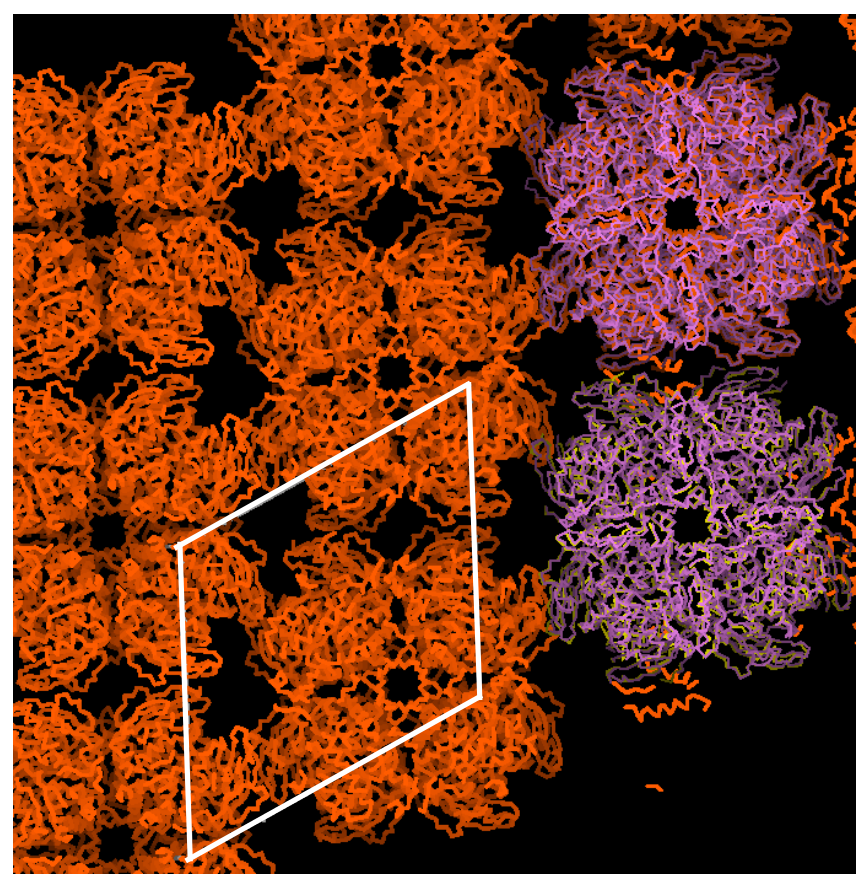

There are only few cases of structural phase transitions reported in the protein crystallography literature. Several systems like lysozyme [16] or factor XIII [17] were reported to undergo the structural phase transition and both phases were described in detail. Two more papers are worth mentioning in this context, in which changes in salt contents causing the phase transition are described $[18,19]$. In those systems the main cause of changes in the structure was associated with the change in the water content of the crystals.

We report here the structural phase transition in the $\mathrm{P} 2{ }_{1}$ crystal form of $G$. sulphuraria RUBISCO. The phase transition in this system leads from two hexadecamers to a single hexadecamer per asymmetric unit. The reason for the structural change is not entirely clear, because the free energy needed to move such large objects ( $\sim 6.6 \mathrm{MDa}$ ) relative to each other is not negligible. We are presently pursuing experiments aiming at clarifying the primary cause of the transition. The literature provides several examples of possible transition mechanisms $[16,17,20]$. Most likely it is the change of water content caused by the freeze-thaw cycle. Indeed, there is a slight change in water content between the two crystal forms of around $1 \%$. The change is minimal but it might be indicative of a change in salt content while the freezing cycle introduces some residual strain.

The observation of the structural phase transition provides interesting implications. Apparently, RUBISCO, a very large macromolecular assembly, can survive the freeze-thaw cycle, providing that the molecule is sufficiently tightly packed to withstand rapid freezing. The hexadecameric molecules interact weakly in the original crystal lattice, thus the reorganization of the lattice does not break internal symmetries of the assembly. The phase transition for such a large macromolecule without loss of diffraction power is rare, testifying to the structural stability of this ancient protein. In conclusion, crystallization of G. sulphuraria RUBISCO will allow us to pursue the details of activation and possibly, further details of the catalytic mechanism. 
Figure 5. Schematic representation of the primary $\mathrm{P} 2_{1}$ lattice (the unit cell in blue) containing two hexadecamers (black and red squares) in the asymmetric unit is shown. The postulated transition of the $2 \mathrm{a}$ crystal form (the blue cell) into the $2 \mathrm{~b}$ crystal form, (the green cell), that proceeds through the hexadecamer rotation is marked by the arrow on the left. The corresponding symmetry elements are in black and dark green. The overlapping elements are in both colors. The relationships presented here are approximate.

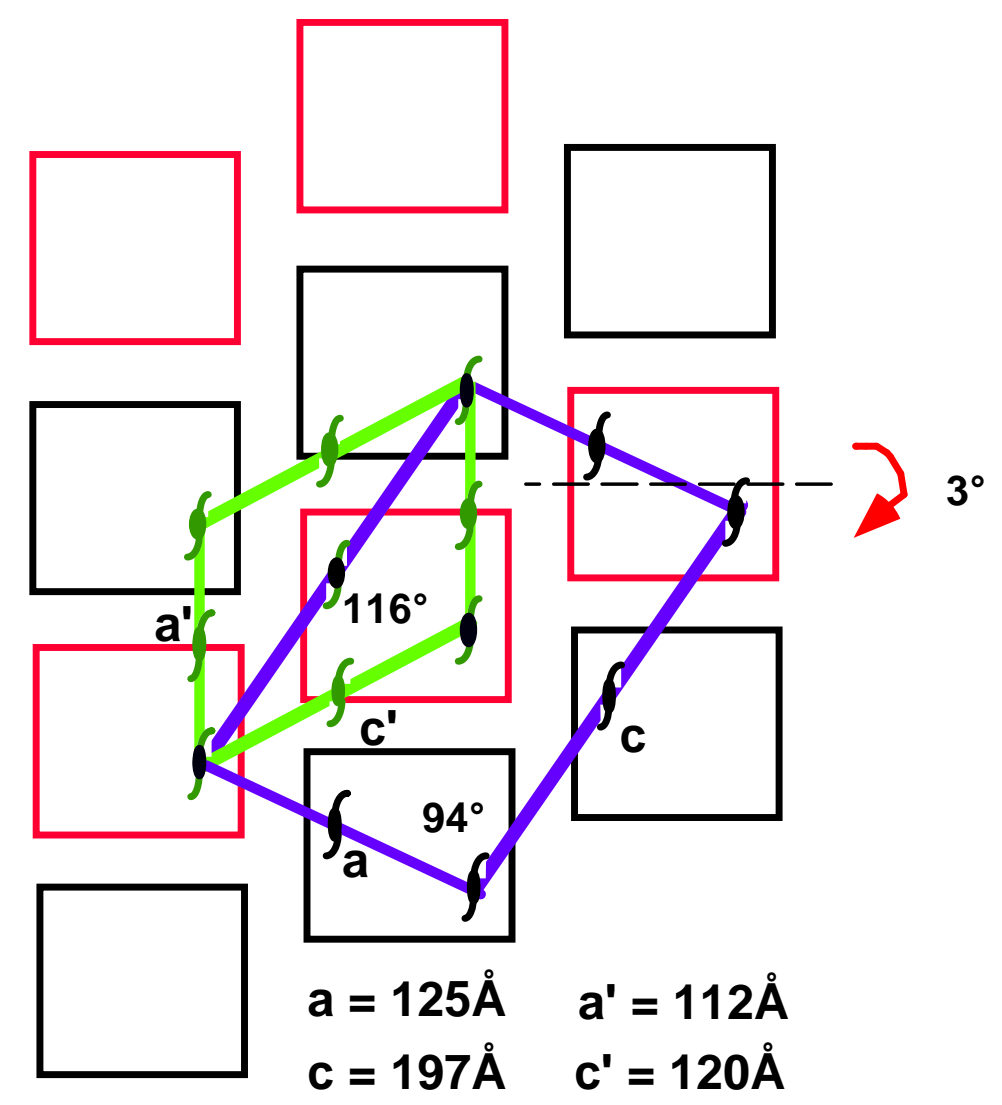

\section{Experimental Section}

\subsection{Materials}

The live cells were gift of Dr. R Troxler (Boston University Medical School). Reagent grade chemicals were purchased from SIGMA.

\subsection{Cell Growth}

The cells were grown in two stages: a glycolytic-dark stage (rapidly dividing) and a photosyntheticlight stage (a greening stage). They were grown in minimal media containing dextrose $(10 \mathrm{~g} / \mathrm{L})$ enriched with a cocktail of micronutrients $(\mathrm{Na}, \mathrm{K}, \mathrm{Mg}, \mathrm{Ca}$ in high $\mu \mathrm{M}$ range, $\mathrm{V}, \mathrm{Mo}, \mathrm{Co}, \mathrm{Cu}, \mathrm{B}$ in low $\mathrm{nM}$ range) at $\mathrm{pH}$ 2. The cells after having grown in dark phase for 10 days were spun down and washed with buffer without dextrose. Then the cells were re-suspended in the same medium without the dextrose and exposed to white light for a period of at least 10 days. The cells when harvested were dark green. A small volume of cells collected after dark stage were mixed with $20 \%$ glycerol and stored frozen for later use at $-80^{\circ} \mathrm{C}$. 


\subsection{Purification}

The cells, collected after the greening stage, were centrifuged at $25,000 \times \mathrm{g}$ for $30 \mathrm{~min}$. The cell pellet was re-suspended in a small volume of the breaking buffer (100 mM Tris, $10 \mathrm{mM} \mathrm{NaCl}, \mathrm{pH} 8)$ and opened by sonication. The cell debris was isolated by centrifugation and the supernatant separated. Subsequently, the soluble proteins were precipitated in 50\% saturated solution of ammonium sulfate. The precipitate was re-suspended and dialyzed against pure water for $24 \mathrm{~h}$. The first chromatographic step was carried out on a brushite column $\left(\mathrm{Ca}_{2} \mathrm{PO}_{4}\right)$. The protein was eluted with the gradient of the phosphate buffer (5 mM to $100 \mathrm{mM}, \mathrm{pH} 8.0$ ). The selected fractions containing colored proteins were precipitated by $50 \%$ AS and dialyzed against pure water. The sample applied on the Biogel-A DEAE ion exchange column was eluted with the gradient of $30 \mathrm{mM}$ to $300 \mathrm{mM} \mathrm{NaCl}$ (pH 7.5). The colored proteins separated from the main non-colored peak, eluted at approximately $100 \mathrm{mM} \mathrm{NaCl}$. The noncolored peak was confirmed by gel electrophoresis to be RUBISCO. The collected protein fractions encompassing major peaks were combined and dialyzed against $0.01 \mathrm{mM}$ phosphate buffer at $\mathrm{pH} 7.5$ and stored at $4{ }^{\circ} \mathrm{C}$.

\subsection{Crystallization}

Concentrated protein $(\sim 11 \mathrm{mg} / \mathrm{mL})$ was crystallized using the hanging drop vapor diffusion method. The ammonium sulfate variable concentration screen was used. Concentrations ranging from $20 \%$ to $60 \% \mathrm{v} / \mathrm{v}$ saturated $\mathrm{AS}$ at three $\mathrm{pH}$ values $(6,7,8)$ were tried. The sample containing $5 \mu \mathrm{L}$ solution of $\sim 11 \mathrm{mg} / \mathrm{mL}$ protein in $0.01 \mathrm{M}$ phosphate buffer mixed with $5 \mu \mathrm{L}$ of $50 \mathrm{mM}$ phosphate buffer $(\mathrm{pH}=7.5)$ containing $55 \% \mathrm{v} / \mathrm{v}$ AS yielded crystals of the form 1 . The crystal of form 1 had a very unusual shape (resembling the optical lens, Figure 1a) and took longer time to grow (approximately 1 month) than the form 2 . The crystals of form 2 were initially obtained by screening with the HAMPTON crystal screen 1. The optimization of crystallization conditions led to the conditions in which the protein suspended in phosphate buffer was mixed with $25 \% \mathrm{v} / \mathrm{v}$ AS and $12 \%$ w/v PEG 4000, yielding large crystals for the crystal form 2 (Figure 1b). The crystals usually grew within 1-2 weeks to typical dimensions of $0.2 \times 0.2 \times 0.3 \mathrm{~mm}$, in the shape of a square pyramid. The crystals of form 2 were harvested, stabilized in the cryoprotectant containing $50 \% \mathrm{w} / \mathrm{v}$ AS and 20\% $\mathrm{w} / \mathrm{v}$ glycerol. They were suspended in a nylon loop and frozen in $\mathrm{N}_{2}$ gas stream at $100 \mathrm{~K}$ for data collection.

\subsection{Data Collection}

The initial crystal characterization and data collection on the crystals of form 1 was carried out on an old multiwire area detector MarcII (Multiwire Area Detector Systems, Poway, CA) at room temperature. The first data set was used for structure solution. The second data set was collected later at $100 \mathrm{~K}$ on the Rigaku RaxisIV++ area detector system mounted on the MicroMax 007 rotating anode generator operated at $40 \mathrm{kV}$ and $20 \mathrm{~mA}$ and processed with HKL2000 [21]. The system is located at the Chemistry Department, University of Texas at El Paso. The data clearly showed high symmetry space group I422 with the diffraction extending to $\sim 2 \AA$ resolution.

The images for the second crystal form showed diffraction to approximately $\sim 2.4 \AA$ resolution (Table 1). The crystal system for the form 2 was monoclinic with the space group $\mathrm{P} 2{ }_{1}$, as determined 
by the analysis of systematic absences. The X-ray data for both crystal phases of form 2 were collected at $100 \mathrm{~K}$. The diffraction data for the form 2 were collected on the same equipment, as described for form 1 . The data were processed with HKL2000.

Table 1. X-ray data collection statistics and contents of the asymmetric unit for I422 and both $\mathrm{P} 2{ }_{1}$ crystal forms.

\begin{tabular}{|c|c|c|c|}
\hline Crystal form & 1 & $2 \mathbf{a}$ & $2 \mathbf{b b}$ \\
\hline Space group & $\mathrm{I} 422$ & $\mathrm{P} 2_{1}$ & $\mathrm{P} 2_{1}$ \\
\hline \multicolumn{4}{|l|}{ Cell dimensions $(\AA)$} \\
\hline$a$ & 136.9, & 124.7 & 111.8, \\
\hline$b$ & 136.9, & 214.6, & 212.3, \\
\hline$c$ & 122.9, & 197.5, & 120.4 \\
\hline$\beta$ & 90.0 & 94.7 & 116.9 \\
\hline Resolution (̊̊) & $37-2.0$ & $34-2.4$ & $34-2.8$ \\
\hline Rmerge* & $0.075(0.41)$ & $0.114(0.52)$ & $0.146(0.39)$ \\
\hline $\mathrm{I} / \sigma(\mathbf{I})$ & $7.2(2.1)$ & $4.2(1.8)$ & $3.7(1.9)$ \\
\hline Redundancy & $6.1(3.1)$ & $2.3(1.7)$ & $1.9(1.7)$ \\
\hline No. reflections & 37654 & 269663 & 99767 \\
\hline Completness (\%) & $89.3(81.0)$ & $85.7(83.2)$ & $79.7(79.2)$ \\
\hline $\begin{array}{l}\text { No. atoms placed in the } \\
\text { asymmetric unit }\end{array}$ & 6224 & 74224 & 37112 \\
\hline
\end{tabular}

*The highest resolution shell in parenthesis.

After the first successful data set collection and data reduction for the monoclinic form, we decided to eliminate the background coming from imperfect cryoprotection. We thawed the crystal, bathed it in fresh stabilizing solution and froze again in the cold stream. To our surprise, we saw a strong yet different diffraction pattern (Figure 2). This new pattern suggested an internal change in the crystal packing. We collected a second data set using the same crystal. The data reduction showed a change in cell dimensions with preservation of monoclinic system (Table 1).

\subsection{Molecular replacement}

The phase problem for both crystal forms was solved using molecular replacement. The structure for the first crystal form was solved by molecular replacement as implemented in CNS [22] using spinach RUBISCO as a template (1AUS). The cell dimensions as well as the symmetry suggested a single large and a single small subunit in the asymmetric unit. The initial solution was unique, taking into account the high symmetry of the lattice with the signal to noise ratio for rotation function of 15.8 sigma remaining peaks around 3 sigma ( $\mathrm{R}$ factor $\sim 0.5$ ). The translation solution was unique and fixed by the internal symmetry of the assembly. The initial placement was followed by a long process of establishing the correct sequence. Only in the last two years with advances in sequencing technologies the unique sequence for Galdieria sulphuraria RUBISCO emerged. The initial fit with Cyanidium caldarium sequence ( $86 \%$ identity, large subunit and $63 \%$ identity, small subunit) showed many mismatches that turned out to be sequence changes between three closely related species of Galdieria partita, Cyanidium caldarium and Galdieria sulphuraria. When the search was conducted with the $G$. 
partita model, the rigid body refinement lowered the $\mathrm{R}$ factor to 0.39 thus confirming the solution obtained before.

The phase problem for the crystal form 2a was solved by MR with G. partita model as a template (1IWA) using the CNS [22]. The cell dimensions suggested that two hexadecameric molecules were present in the asymmetric unit. The self-rotation function suggested that the fourfold axis of the hexadecamers were close to the $\mathrm{Y}$ axis while both hexadecamers were rotated against each other (Figure 3). Indeed, two hexadecamers were located in the asymmetric unit with the resulting R factor of 0.49 . The initial rotation function produced eight peaks approximately 30 sigma above the noise level, while the next peaks on the list were at the level of 5 sigma. All those peaks were equivalent by non-crystallographic 422 symmetry. We are presently continuing the refinement of the initial model because the $\sim 10,000$ amino acid contents of the asymmetric unit makes it a formidable technical task. We are working on implementing effective refinement strategies.

A similar process to solve the phase problem was carried out for the second monoclinic form using the same model of $G$. partita RUBISCO. As suggested by the cell dimensions, only a single hexadecamer was located in the asymmetric unit. The highest peaks were again above 30 sigma and $\mathrm{R}$ $\sim 0.45$, indicating the correctness of the solution. The self-rotation function clearly showed that a major fourfold axis was positioned as in form $2 \mathrm{a}$ along the $\mathrm{Y}$ direction while the perpendicular two-fold symmetry axis was aligned with the $\mathrm{X}$ direction. The simplification of the self-rotation function combined with detected directions of two-fold axes when compared to form $2 \mathrm{a}$ was consistent with the presence of a single hexadecamer in the asymmetric unit.

\section{Acknowledgements}

We would like to thank Dr. R. Troxler for live samples of red algae.

\section{References and Notes}

1. Zelitch, I. Pathways of carbon fixation in green plants. Ann. Rev. Biochem. 1975, 44, 123-45

2. Pereto, J. G.; Velasco, A. M.; Becerra, A.; Lazcano, A. Comparative biochemistry of CO2 fixation and the evolution of autotrophy. Int. Microbiol. 1999, 2, 3-10.

3. Newman, J.; Gutteridge, S. Structure of an effector-induced inactivated state of ribulose 1,5bisphosphate carboxylase/oxygenase: the binary complex between enzyme and xylulose 1,5bisphosphate. Structure 1994, 2, 495-502.

4. Andersson, I. Large structures at high resolution: the 1.6 A crystal structure of spinach ribulose1,5-bisphosphate carboxylase/oxygenase complexed with 2-carboxyarabinitol bisphosphate. $J$. Mol. Biol. 1996, 259, 160-74.

5. Zhang, K.Y.; Cascio, D.; Eisenberg, D. Crystal structure of the unactivated ribulose 1,5bisphosphate carboxylase/oxygenase complexed with a transition state analog, 2-carboxy-Darabinitol 1,5-bisphosphate. Protein Sci. 1994, 3, 64-9.

6. Sugawara, H.; Yamamoto, H.; Shibata, N.; Inoue, T.; Okada, S.; Miyake, C.; Yokota, A.; Kai, Y. Crystal structure of carboxylase reaction-oriented ribulose 1,5-bisphosphate carboxylase/oxygenase from a thermophilic red alga, Galdieria partita. J. Biol. Chem. 1999, 274, 15655-15661.

7. Cleland, W.W.; Andrews, T.J.; Gutteridge, S.; Hartman, F.C.; Lorimer, G.H. Mechanism of 
Rubisco: The carbamate as general base. Chem. Rev. 1998, 98, 549-561

8. Zhang, N.; Portis, A.R.Jr. Mechanism of light regulation of Rubisco: a specific role for the larger Rubisco activase isoform involving reductive activation by thioredoxin-f. Proc. Natl. Acad. Sci. U. S. A. 1999, 96, 9438-43.

9. Sekbach, J.; Fredrick, J.F. A primaeval alga bridging the blue-green and the red algae: further biochemical and ultrastructure studies of Cyanidium caldarium with special reference to the plastid membranes. Microbios 1980, 29, 135-47

10. Valentin, K.; Zetsche, K. Structure of the Rubisco operon from the unicellular red alga Cyanidium caldarium: evidence for a polyphyletic origin of the plastids. Mol. Gen. Genet. 1990, 222, 425-30.

11. Spreitzer, R. J.; Salvucci, M. E. Rubisco: structure, regulatory interactions, and possibilities for a better enzyme. Ann. Rev. Plant. Biol. 2002, 53, 449-75.

12. Uemura, K.; Anwaruzzaman, M.; Miyachi, S.; Yokota, A. Ribulose-1,5-bisphosphate carboxylase/oxygenase from thermophilic red algae with a strong specificity for CO2 fixation. Biochem. Biophys. Res. Commun. 1997, 233, 568-71.

13. Ford, T. W. Ribulose 1,5-bisphosphate carboxylase from the thermophilic, acidophilic alga, Cyanidium caldarium (Geitler). Purification, characterization and thermostability of the enzyme Biochem. Biophys. Acta 1979, 569, 239-248

14. Curmi, P.M.; Cascio, D.; Sweet, R.M.; Eisenberg, D.; Schreuder, H. Crystal structure of the unactivated form of ribulose-1,5-bisphosphate carboxylase/oxygenase from tobacco refined at 2.0A resolution. J. Biol. Chem. 1992, 267, 16980-9,

15. Okano, Y.; Mizohata, E.; Xie, Y.; Matsumura, H.; Sugawara, H.; Inoue, T.; Yokota, A.; Kai, Y. $\mathrm{X}$-ray structure of Galdieria Rubisco complexed with one sulfate ion per active site. FEBS Lett. 2002, 527, 33-6.

16. Harata, K.; Akiba, T. Phase transition of triclinic hen egg-white lysozyme crystal associated with sodium binding. Acta Crystallogr. D 2004, 60, 630-7.

17. Weiss, M. S.; Hilgenfeld, R. Dehydration leads to a phase transition in monoclinic factor XIII crystals. Acta Crystallogr D 1999, 55, 1858-62.

18. Declerq, J.P.; Evrard, C. A twinned monoclinic crystal form of human peroxiredoxin 5 with eight molecules in the asymmetric unit. Acta Crystallogr. D 2001, 57, 1829-1835.

19. Dauter, Z.; Li, M.; Wlodawer, A. Practical experience with the use of halides for phasing macromolecular structures: a powerful tool for structural genomics. Acta Crystallogr. D 2001, 57, 239-249

20. Skrzypczak-Jankun, E.; Bianchet, M. A.; Amzel, M. L.; Funk, M. O. Jr. Flash-freezing causes a stress-induced modulation in a crystal structure of soybean lipoxygenase Acta Crystallogr. D 1996, 52, 959-65.

21. Otwinowski, Z.; Minor W. Processing of X-Ray Diffraction Data Collected in Oscillation Mode. Method. Enzymol. 1997, 276, 307-326

22. Brunger, A. T.; Adams, P. D.; Clore, G. M.; DeLano, W. L.; Gros, P.; Grosse-Kunstleve, R. W.; Jiang, J. S.; Kuszewski, J.; Nilges, M.; Pannu, N. S.; Read, R. J.; Rice, L. M.; Simonson, T.; Warren, G. L. Crystallography \& NMR system: A new software suite for macromolecular structure determination. Acta Crystallogr. D . 1998, 54, 905-21.

(C) 2007 by MDPI (http://www.mdpi.org). Reproduction is permitted for noncommercial purposes. 